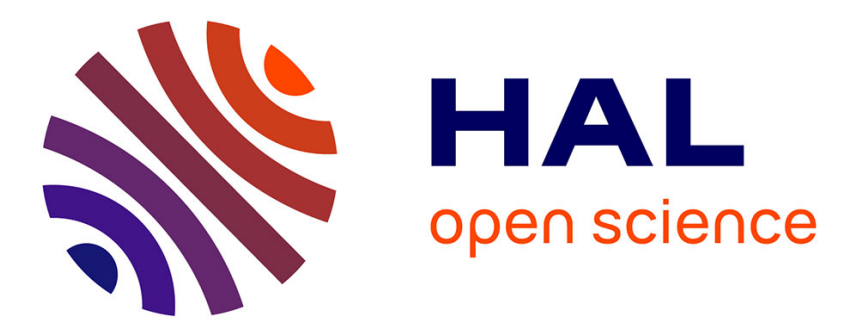

\title{
Stochastic spectral-spatial permutation ordering combination for nonlocal morphological processing \\ Olivier Lézoray
}

\section{To cite this version:}

Olivier Lézoray. Stochastic spectral-spatial permutation ordering combination for nonlocal morphological processing. International Conference on Systems, Signals and Image Processing, IEEE, May 2017, Poznan, Poland. hal-01526273

\section{HAL Id: hal-01526273 https://hal.science/hal-01526273}

Submitted on 22 May 2017

HAL is a multi-disciplinary open access archive for the deposit and dissemination of scientific research documents, whether they are published or not. The documents may come from teaching and research institutions in France or abroad, or from public or private research centers.
L'archive ouverte pluridisciplinaire HAL, est destinée au dépôt et à la diffusion de documents scientifiques de niveau recherche, publiés ou non, émanant des établissements d'enseignement et de recherche français ou étrangers, des laboratoires publics ou privés. 


\title{
Stochastic spectral-spatial permutation ordering combination for nonlocal morphological processing
}

\author{
Olivier Lézoray \\ Normandie Univ, UNICAEN, ENSICAEN, CNRS, GREYC, 14000 Caen, France
}

\begin{abstract}
The extension of mathematical morphology to multivariate data has been an active research topic in recent years. In this paper we propose an approach that relies on the consensus combination of several stochastic permutation orderings. The latter are obtained by searching for a smooth shortest path on a graph representing an image. The construction of the graph can be based on both spatial and spectral information and naturally enables patch-based nonlocal processing.
\end{abstract}

\section{INTRODUCTION}

Mathematical morphology (MM) is a non linear image processing framework with many applications in filtering, segmentation and classification [1]. The construction of morphological operators relies on complete lattices [2] that impose the need of an ordering relationship between the elements to be processed. A complete lattice $(\mathcal{T}, \leq)$ is a non-empty set equipped with a total ordering relation, such that every nonempty subset $\mathcal{P}$ of $\mathcal{T}$ has a lower bound $\wedge \mathcal{P}$ and an upper bound $\vee \mathcal{P}$. In this context, images are modeled by functions mapping their domain space $\Omega$, into a complete lattice $\mathcal{T}$, and are expressed as $f: \Omega \subset \mathbb{Z}^{l} \rightarrow \mathcal{T} \subset \mathbb{R}^{n}$ where $l$ is the image dimension, $n$ the number of channels, and $\mathcal{T}$ is a non-empty set of vectors. If MM is well defined for gray scale functions, there exists no general admitted extension that permits to perform morphological operations on vectors since there is no natural ordering of vectors [3].

To cope with this well-known issue, several approaches have been proposed (see [4], [5], [6] and references therein). The most recently proposed ones define an ordering relation between the vectors of a set $\mathcal{T}$ with the use of $h$-orderings [7]. This corresponds to defining a surjective transform $h$ from $\mathcal{T}$ to $\mathcal{L}$ where $\mathcal{L}$ is a complete lattice equipped with the conditional total ordering [7]. We refer to $\leq_{h}$ as the $h$ ordering given by:

$$
\begin{aligned}
& h: \mathcal{T} \rightarrow \mathcal{L} \text { and } \mathbf{v} \rightarrow h(\mathbf{v}), \forall\left(\mathbf{v}_{i}, \mathbf{v}_{j}\right) \in \mathcal{T} \times \mathcal{T} \\
& \mathbf{v}_{i} \leq_{h} \mathbf{v}_{j} \Leftrightarrow h\left(\mathbf{v}_{i}\right) \leq h\left(\mathbf{v}_{j}\right) .
\end{aligned}
$$

Then, $\mathcal{T}$ is no longer required to be a complete lattice, since the ordering of $\mathcal{T}$ can be induced upon $\mathcal{L}$ by means of $h$ [4]. When $h$ is bijective, this corresponds to defining a space filling curve [8] or equivalently a rank transform [9].

Most approaches for ordering vectors (including $h$ orderings) have focused on building an ordering on the definition domain of the spectral vectors of the set $\mathcal{T}$, without taking into account how the corresponding vectors are located in the image support. This provides a general framework, but it can be more interesting to find the best order for a given image rather than looking for the best order in general, as argued in [10]. Recent $h$-ordering approaches have searched to define total orders that are adapted to only the set $\mathcal{T}$ of spectral vectors of an image [5]. In addition, this adaptation can consider also the spatial organization of the spectral vectors in the image. Indeed, some vectors of the set $\mathcal{T}$ will never have to be compared since they will not appear within a given structuring element. Consequently, having an ordering that considers spatial proximity in addition to spectral proximity can be of strong interest. Some works have considered that trend of spectral-spatial ordering with the use of hierarchical clustering [11], binary partition tree [12] or local hamiltonian path [13].

In this paper we propose a new spatial-spectral ordering based on the combination of several stochastic orderings (also called permutations) that each construct a global hamiltonian path on an image. The paper is organized as follows. In section II we present the construction of a global hamiltonian path on an image with 1D permutation ordering on grid-graphs. We then expose in section III how this ordering can take into account both spatial and spectral proximity with the use of nonlocal graphs. Since the proposed ordering depends on a starting pixel, several orders are obtained that are combined using a consensus approach in section IV. Last sections present results and conclude.

\section{ONE DIMENSIONAL PERMUTATION ORDERING}

We consider that the domain $\Omega$ of the image is a grid graph $\mathcal{G}=(\mathcal{V}, \mathcal{E})$ where vertices $\mathcal{V}=\left\{v_{1}, \ldots, v_{m}\right\}$ correspond to pixels and edges $e_{i j}=\left(v_{i}, v_{j}\right)$ connect vertices with 8adjacency. The notation $v_{i} \sim v_{j}$ is used to denote two adjacent vertices. With this definition, images are represented as graphs signals [14] that associate vectors to vertices and are defined by the mapping $f: \mathcal{G} \rightarrow \mathcal{T} \subset \mathbb{R}^{n}$ where $\mathcal{T}$ is a non-empty set of vectors (we will consider only RGB color vectors, i.e., $n=3$ ). To each vertex $v_{i} \in \mathcal{G}$ is associated a vector $\mathbf{v}_{i}=f\left(v_{i}\right)$. The set $\mathcal{T}=\left\{\mathbf{v}_{1}, \cdots, \mathbf{v}_{m}\right\}$ denotes the vectors associated to all the vertices of the graph (with a row-major numbering of the image graph vertices). We will use the notation $\mathcal{T}[i]=\mathbf{v}_{i}$ to denote the $i$-th element of a set.

To construct an image-adaptive $h$-ordering, we propose to construct a space-filling curve [8] on the grid graph $\mathcal{G}$. This corresponds to the constuction of a global hamiltonian path on all the image: a path that goes through all the vertices of the grid graph and traverses each vertex only one. This was 
previously explored in [13] but with local hamiltonian paths. However, with such a local ordering, the associated dilation and erosion are not strictly speaking morphological operators since they do not commute with the sup. We consider a more general approach and build an order for all the set $\mathcal{T}$ in the form of a global hamiltonian path.

Given the set $\mathcal{T}$, the construction of an hamiltonian path amounts to define a sorted permutation $\mathcal{P}=\mathbf{P} \mathcal{T}$ of the vectors of $\mathcal{T}$ with $\mathbf{P}$ a permutation matrix of size $m \times m$. Let $\sigma$ be a permutation of the index set $\mathcal{I}=\{1, \cdots, m\}$. If $\sigma(i)=j$, then $\mathbf{P}_{i j}=1$ and 0 otherwise. The induced permutation is $\mathcal{P}=\mathbf{P} \mathcal{T}=\left\{\mathbf{v}_{\sigma^{-1}(1)}, \cdots, \mathbf{v}_{\sigma^{-1}(m)}\right\}$ where $\left(\sigma^{-1} \circ \sigma\right)$ is the identity. Any permutation is not of interest, and spatial and spectral constraints have to be taken into account [10]. To this end we consider the construction of a smooth permutation. The smoothness of an ordered set is expressed by the Total Variation of its elements:

$$
\|\mathcal{T}\|_{T V}=\sum_{i=1}^{m-1}\left\|\mathbf{v}_{i}-\mathbf{v}_{i+1}\right\|
$$

The optimal permutation operator $\mathbf{P}$ can be obtained by minimizing the total variation of $\mathbf{P} \mathcal{T}$ :

$$
\mathbf{P}^{*}=\underset{\mathbf{P}}{\arg \min }\|\mathbf{P} \mathcal{T}\|_{T V}
$$

This optimization problem provides a permutation such that the corresponding hamiltonian path is the shortest one and is equivalent to solve the traveling salesman problem. This problem being too computationally demanding for large sets, it can be solved using a randomized version of nearest neighbors heuristics presented in [15], [16]. We recall the principle of this algorithm in Algorithm 1. This algorithm starts from an arbitrary vertex and continues by finding the two nearest unexplored neighbor vertices and choosing one of them at random
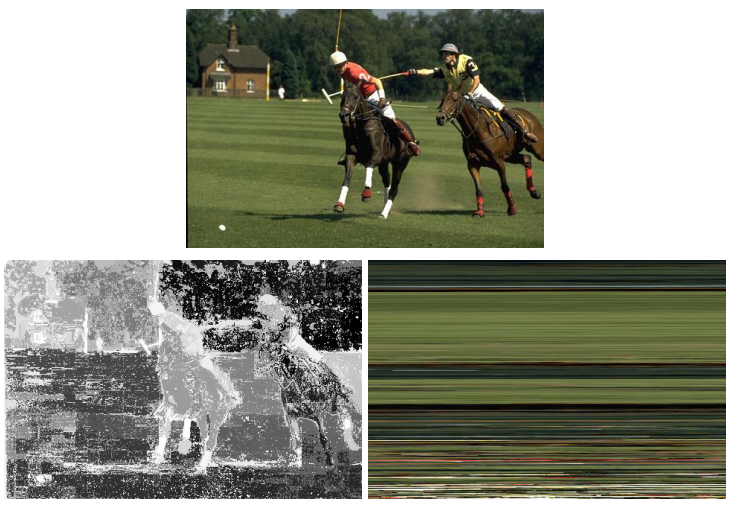

Fig. 1. Example of permutation ordering. First line presents the original image, second line an obtained index $I$ and its associated permutation $\mathcal{P}$.

After the construction of the permutation, we define the $h$ ordering from the constructed permutation as $h\left(\mathbf{v}_{i}\right)=\sigma(i)$ and this defines the complete lattice $\left(\mathcal{T}, \leq_{h}\right)$. Given a graph signal $f: \mathcal{G} \rightarrow \mathcal{T}$, a new representation is obtained in the form of the pair $(I, \mathcal{P})$ with $I\left(v_{i}\right)=\sigma(i)$. When a graph signal is

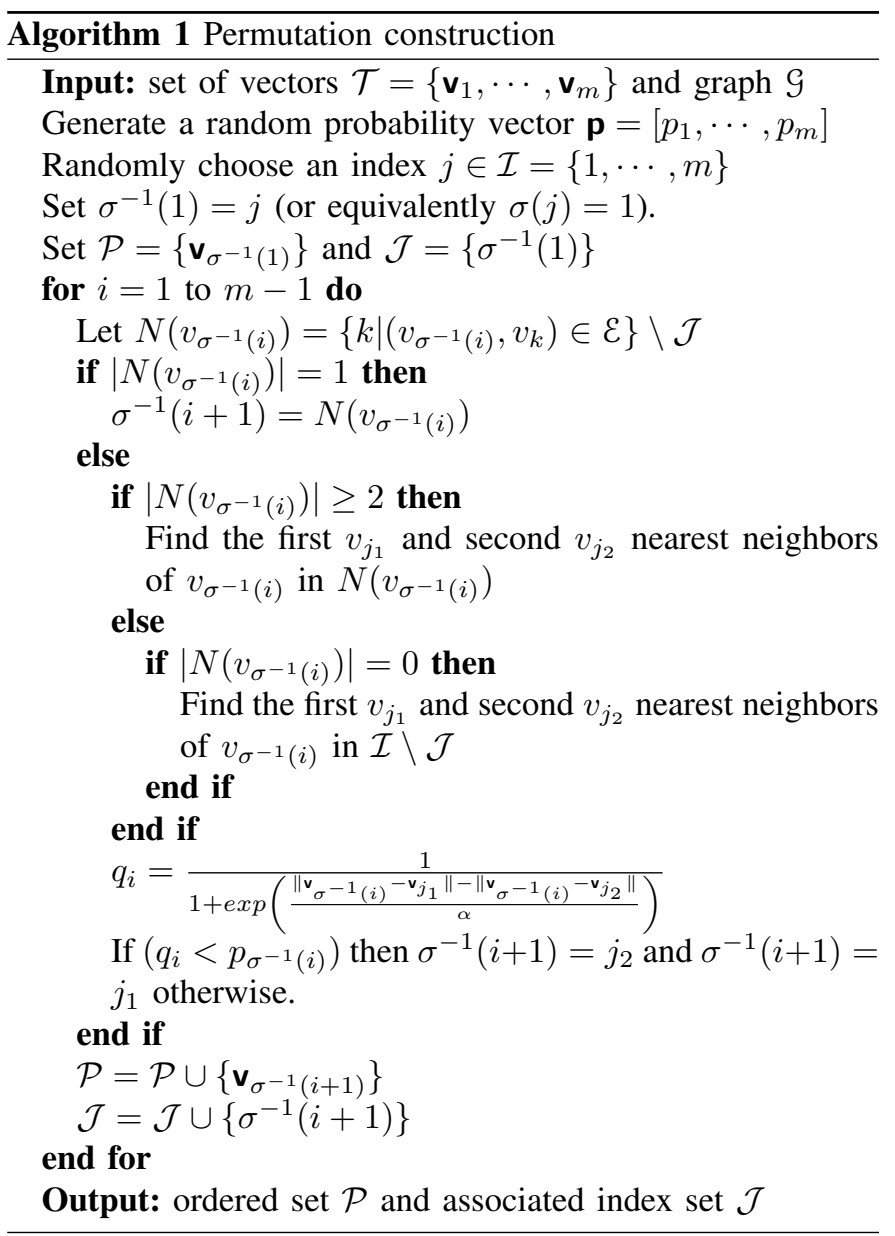

encoded in this way, the spectral information is not directly carried by the index $I$, but is stored in a separate piece of data called a palette: the set $\mathcal{P}$ of sorted vectors. Figure 1 presents the obtained permutation and index from an original image. A drawback of the described ordering is its greediness nature. Indeed, as can be seen in Figure 1, the last part of the permutation is not smooth.

The original graph signal $f$ can be directly recovered since $f\left(v_{i}\right)=\mathcal{P}\left[I\left(v_{i}\right)\right]=\mathcal{T}[i]=\mathbf{v}_{i}$. As exposed in [5], [14], from such a representation, morphological operations can be defined and applied to the graph signal. The erosion and dilation of a graph signal $f$ at vertex $v_{i} \in \mathcal{G}$ by a structuring element $B_{k} \subset \mathcal{G}$ are defined as:

$$
\begin{gathered}
\epsilon_{B_{k}}(f)\left(v_{i}\right)=\left\{\mathcal{P}\left[\wedge I\left(v_{j}\right)\right], v_{j} \in B_{k}\left(v_{i}\right)\right\} \\
\delta_{B_{k}}(f)\left(v_{i}\right)=\left\{\mathcal{P}\left[\vee I\left(v_{j}\right)\right], v_{j} \in B_{k}\left(v_{i}\right)\right\} .
\end{gathered}
$$

A structuring element $B_{k}\left(v_{i}\right)$ of size $k$ defined at a vertex $v_{i}$ corresponds to the set of vertices that can be reached from $v_{i}$ in $k$ walks.

\section{SPECTRAL-SPATIAL PERMUTATION ORDERING}

In Algorithm 1, there is no restriction on the considered graph. However, in section II, we have restricted ourselves to 8-adjacency grid graphs, but there is no reason to do so. 
In this section we consider several possible graphs for the construction of the permutation. The graph can use only spatial or spectral information, or both.

- 8-adjacency grid graph (denoted $\left.\mathcal{G}_{0}\right)$ : this graph construction connects each vertex to its 8 spatially closest nearest neighbors.

- $B$-adjacency graph (denoted $\mathcal{G}_{B}$ ): this graph construction connects each vertex $v_{i}$ to all the vertices contained in a square box of size $(2 B+1) \times(2 B+1)$ around $v_{i}$.

- $K$-Nearest Neighbor graph (denoted $\mathcal{G}_{K}^{s}$ ): this graph construction connects each vertex $v_{i}$ to its $K$ nearest neighbors (in terms of spectral distance) within the set of all vertices.

The first two kinds of graphs $\mathcal{G}_{0}$ and $\mathcal{G}_{B}$ consider only the spatial proximity information to connect vertices whereas the graph $\mathcal{G}_{K}^{s}$ considers only the spectral proximity information and is a nonlocal graph. It can be interesting to construct graphs that combine the use of both spatial and spectral proximity to infer the set of edges. For the graph $\mathcal{G}_{K}^{s}$ it is even recommended since its construction cannot ensure that the graph is connected and Algorithm 1 will not be able to reach all vertices. This can be easily obtained by considering the union of the set of edges from the previously considered graphs. Given such a graph construction, with Algorithm 1, a spectral-spatial permutation ordering is then obtained. This can be very interesting since both spatial and spectral proximities are taken into account to build the permutation and this can reduce computation complexity and ensure that relevant nearest neighbors are found. In addition, the spectral proximity used to determine the nearest neighbors within the set of adjacent vertices can consider any distance measure. In particular, instead of using a classical $L_{2}$ distance between the vectors $\mathbf{v}_{i}$ associated to vertices, one can consider patches $\mathbf{p}_{i}^{w}=\left(f\left(x_{i}+t\right), \forall t \in[-w / 2, w / 2]^{2}\right)^{T}$ of size $w \times w$ to obtain a smoother ordering.

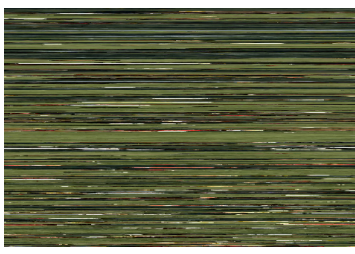

$\mathcal{G}_{0}$

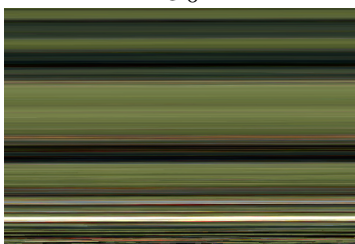

$\mathcal{G}_{0} \cup \mathcal{G}_{20}^{s}$ with $\mathbf{v}_{i}$

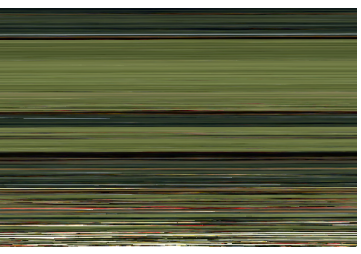

$\mathcal{G}_{10}$

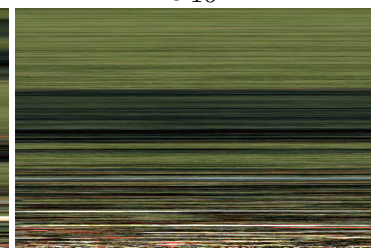

$\mathcal{G}_{0} \cup \mathcal{G}_{20}^{s}$ with $\mathbf{p}_{i}^{3}$
Fig. 2. Illustration of the influence of graph construction on the obtained permutation ordering.

Figure 2 presents several permutation orderings obtained with different graphs. It can be seen that with $\mathcal{G}_{0}$ the permutation is not very smooth and the result obtained with a box-constrained graph is much better (graph $\mathcal{G}_{10}$ ). Finally the permutation ordering can be very different by considering both spatial and spectral information. Last line of Figure 2 illustrates this with two permutations built on the union of a grid graph $\mathcal{G}_{0}$ and a nearest neighbor graph $\mathcal{G}_{20}^{s}$ with measuring spectral similarity either with color vectors $\mathbf{v}_{i}$ or patches $\mathbf{p}_{i}^{3}$ of size $3 \times 3$.

\section{CONSENSUS ORDERING}

Algorithm 1 is a randomized process that starts from an arbitrary vertex of the graph. As a consequence, at each run of the permutation ordering, if a different starting vertex is chosen, a different result is obtained. In [15], [16], the authors have made the most of this by using several permutations for denoising purposes. We propose to proceed differently and to combine several permutation orderings into a single one. We denote as $h_{i}$ a given ordering and we consider a set of $l$ available orderings. The principle of combining several orderings (also called ranks or permutations) into a single one is known as ranking aggregation, the aim of which being to provide a consensus ranking [17]. Finding the consensus permutation can be formulated as an optimization problem, which is usually NP-hard [18] and heuristic ranking aggregation methods have been proposed. In this paper we consider the following ones [17].

a) Instant-Runoff aggregation: this aggregation method determines the ranking position of the item with the fewest votes and iterates on the resting items. The process is as follows. One begins by counting the first place votes according to the rankings with respect to the remaining items with $R\left(\mathbf{v}_{j}\right)=\sum_{i=1}^{l} \delta_{i}\left(\mathbf{v}_{j}\right)$ where $\delta_{i}\left(\mathbf{v}_{j}\right)$ indicates the first place votes according to permutation $h_{i}$ and is defined by

$$
\delta_{i}\left(\mathbf{v}_{j}\right)=\left\{\begin{array}{l}
1 \text { if } \mathbf{v}_{j} \geq_{h_{i}} \mathbf{v}_{k}, \forall k \neq j \\
0 \text { otherwise }
\end{array}\right.
$$

The item with the fewest votes is assigned the lowest position in the obtained consensus rank.

b) Borda count: this methods determines the ranking position of all items simultaneously. It assigns each item $\mathbf{v}_{\mathbf{j}}$ $(j \in \mathcal{I})$ a score $B^{i}\left(\mathbf{v}_{j}\right)$ based on the positions provided by the permutations $h_{i}(i=1, \ldots, l)$ :

$$
B^{i}\left(\mathbf{v}_{j}\right)=1-\frac{h_{i}\left(\mathbf{v}_{j}\right)-1}{m}
$$

According to the scores, Borda count generates a final score using mean aggregation $B S\left(\mathbf{v}_{j}\right)=\frac{1}{l} \sum_{k=1}^{l} B^{k}\left(\mathbf{v}_{j}\right)$. The fused ranking is obtained by sorting the aggregated scores of items.

c) Weighted Borda count: we propose to modify the Borda count score in order to take into account the smoothness of each permutation. Indeed, in our case, we aggregate permutation orderings that represents smooth shortest paths and we would like to obtain a consensus permutation that is also smooth. To do so, we consider a weighted score defined by

$$
B_{s}^{i}\left(\mathbf{v}_{j}\right)=B^{i}\left(\mathbf{v}_{j}\right) \times \nabla \mathcal{P}_{i}\left(\mathbf{v}_{j}\right)
$$



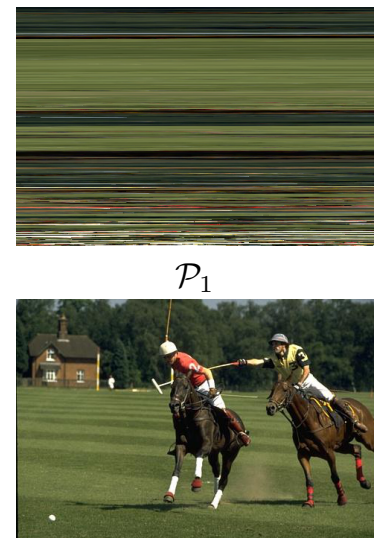

Original image

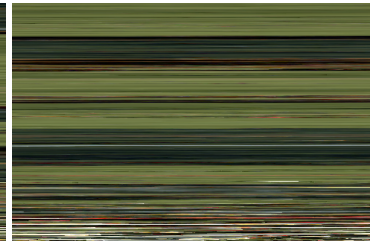

$\mathcal{P}_{2}$

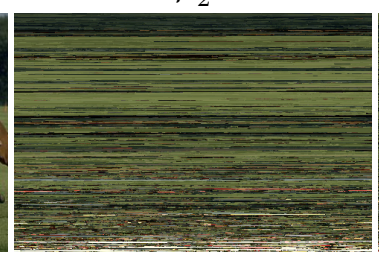

Instant-Runoff $\mathcal{P}$

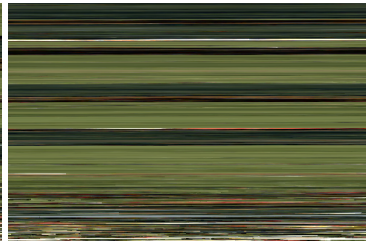

$\mathcal{P}_{3}$

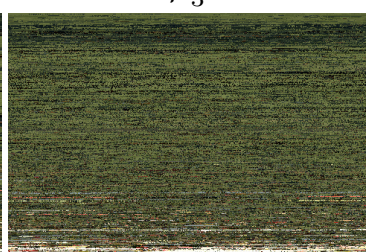

Borda count $\mathcal{P}$

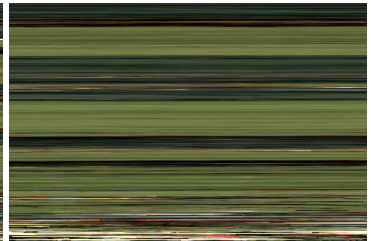

$\mathcal{P}_{4}$

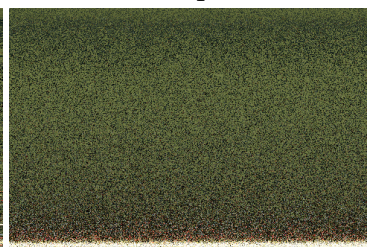

Weighted borda count $\mathcal{P}$ Weighted borda count $I$

Fig. 3. Consensus combination of different stochastic permutations.

where $\nabla \mathcal{P}_{i}\left(\mathbf{v}_{j}\right)$ is a 1D-gradient measured on the permutation ordering from the right and left neighbors of $\mathbf{v}_{j}$ in $\mathcal{P}_{i}$

Figure 3 illustrates the principle of permutation ordering combination. Given an original image (appears on last row, first column), five different permutation orderings $\left(\mathcal{P}_{1}, \cdots, \mathcal{P}_{5}\right)$ have been generated using different starting vertices. They are shown in first row of Figure 3. The obtained permutations can be very different from one to another due to the stochastic nature of Algorithm 1. Last row of Figure 3 presents the different consensus permutations that can be obtained with Instant-Runoff, Borda count or weighted Borda count. As it can be seen, the borda count provides a smoother consensus than the Instant-Runoff, and this is enhanced with the proposed weighted version of the Borda count.

\section{RESULTS}

Now that we have a method that enables the generation of a consensus permutation from several stochastic ones, we are in position to use the obtained permutation to perform morphological processing of images. In this section we investigate the influence of the different parameters of the approach: the combination method, the graph under construction, the spectral proximity measure. Figure 4 presents an illustration of the influence of these parameters with the result of a closing operation $\phi=\epsilon \delta$ with a $5 \times 5$ square structuring element. The number of combined permutation is 5 and the considered graph is $\mathcal{G}_{10}$.

As shown in the two first rows of Figure 4, there is few differences between Instant-Runoff and Borda count consensus combination, and closings with these combination methods are not able to well preserve the object edges in the image. This is not the case for weighted Borda count where the edges are preserved and well-delineated, as expected for a morphological processing. Last row of Figure 4 presents the influence of the graph construction. This corresponds to the weighted Borda count combination of 5 spatial-spectral stochastic permutations with the use of either color vectors or $5 \times 5$ patches for spectral proximity measurement. As it can be seen the combination of both spatial and spectral can be
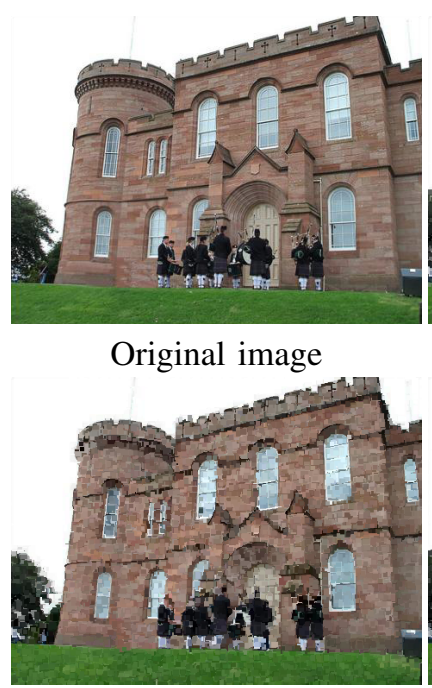

Borda count

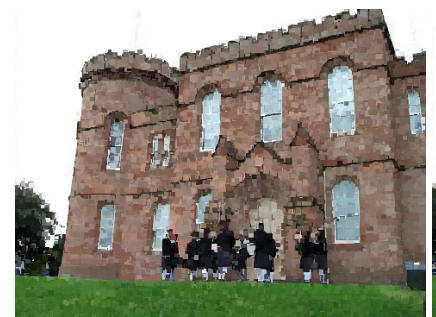

$\mathcal{G}_{10} \cup \mathcal{G}_{20}^{s}$ with $\mathbf{v}_{i}$

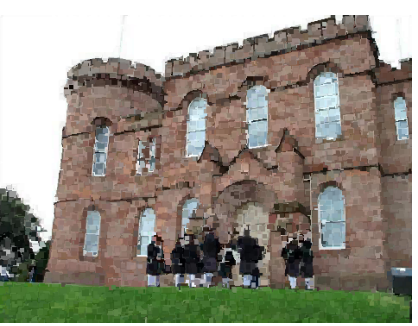

Instant-Runoff

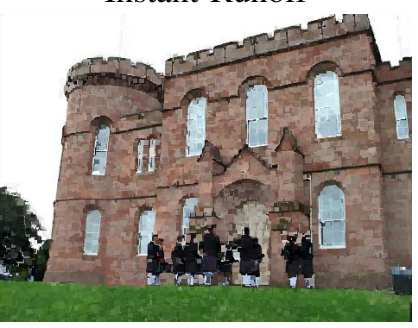

Weighted Borda count

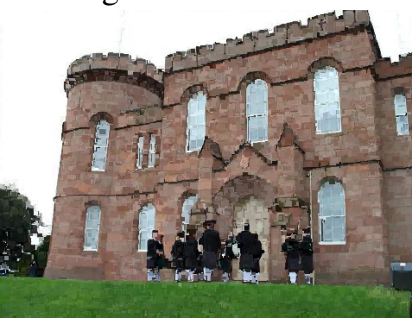

$\mathcal{G}_{10} \cup \mathcal{G}_{20}^{s}$ with $\mathbf{p}_{i}^{5}$
Fig. 4. Influence of the parameters on a stochastic permutation closing with the proposed approach.

interesting but only when the proximity measure is accurate enough. Indeed, with color vectors the obtained result does not differ much from the one with only spatial constraints for the graph construction. Inversely, the use of patches has enabled to enhance the result that is much more contrasted, and better preserves edges and texture. This offers a new alternative for nonlocal morphological processing of images. This is all the more visible in Figure 5 where a closing is performed (with a $5 \times 5 \mathrm{SE}$ ) on an image with a local processing based on color 
vectors (with a $\mathcal{G}_{10}$ graph) and a nonlocal processing based on patches (with a $\mathcal{G}_{10} \cup \mathcal{G}_{20}^{s}$ graph).

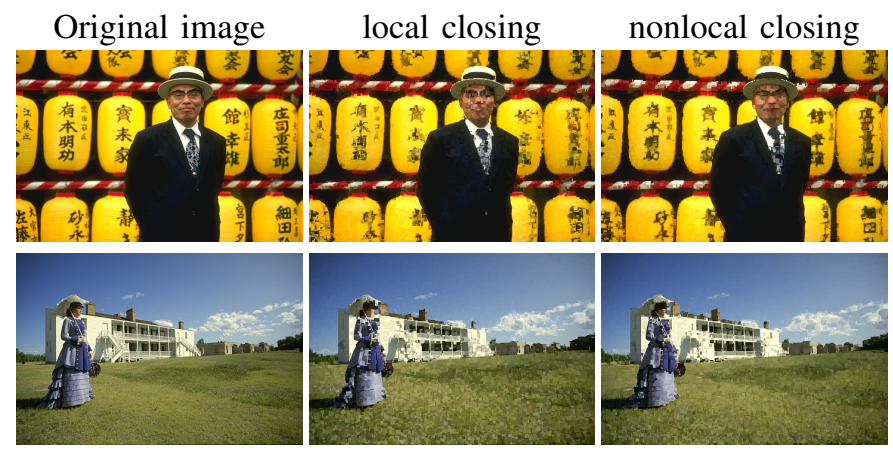

Fig. 5. Interest of patch-based processing with, for each row and from left to right, the original image, a local and a nonlocal closing.

Finally we compare the proposed nonlocal processing with the approach of [19] (denoted NL MM). Figure 6 presents the results of classical MM, non local morphology with [19] and with our approach. For nonlocal processing $5 \times 5$ patches are considered. As it can be seen, the approach of [19] does not provide accurate results and acts more like a gamma correction and artificially enhances background noise. This can be seen on the gradient image that does not correspond to the expected result of a gradient operator. In contrast, our approach has an interpretation that is similar to the classical morphological operators while leveraging the simplification effect to preserve important edges and textures in the image. The gradient image illustrates this and enables a much more accurate extraction of the edges.

\section{CONCLUSION}

This paper has introduced a novel approach for nonlocal morphological processing of images. Several stochastic permutation orderings are first constructed so as to obtain smooth paths on a graph. The construction of the graph can benefit from both spatial and spectral information and enables to obtain permutation ordering that rely on patches instead of single colors. The permutation orderings are combined into a single one using a weighted borda count approach to produce a smooth permutation ordering consensus.

\section{REFERENCES}

[1] J. Serra, Image Analysis and Mathematical Morphology, Academic Press, Inc., Orlando, FL, USA, 1983.

[2] C. Ronse, "Why mathematical morphology needs complete lattices," Signal Processing, vol. 21, no. 2, pp. 129-154, 1990.

[3] V. Barnett, "The ordering of multivariate daa," J R STAT SOC SER $A-G$, vol. 139 , no. 3, pp. 318-355, 1976.

[4] E. Aptoula and S. Lefèvre, "Multivariate mathematical morphology applied to colour image analysis," in Multivariate image processing: methods and applications, C. Collet, J. Chanussot, and K. Chehdi, Eds., pp. 303-337. ISTE - John Wiley, 2009.

[5] O. Lézoray, "Complete lattice learning for multivariate mathematical morphology," J VIS COMMUN IMAGE R, vol. 35, pp. 220-235, 2016.

[6] S. Velasco-Forero and J. Angulo, "Mathematical morphology for vector images using statistical depth," in ISMM, 2011, vol. LNCS 6671, pp. $355-366$.

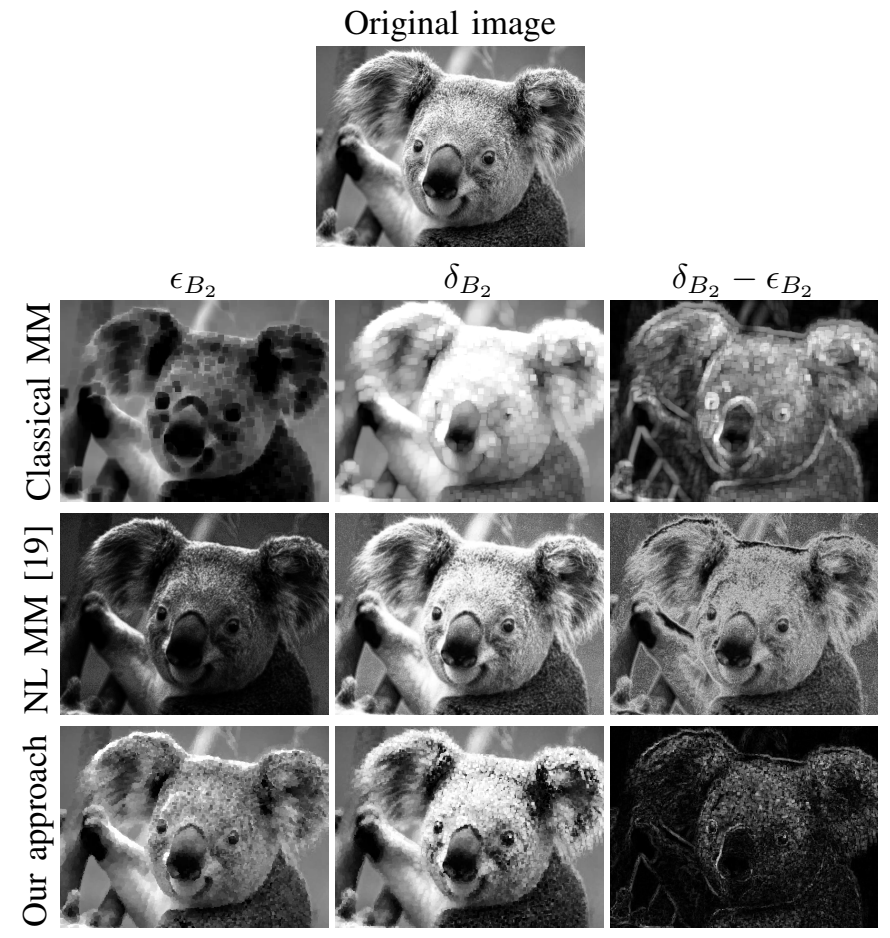

Fig. 6. Comparison between classical MM, NL MM [19], and our approach with a $5 \times 5$ SE (one approach per line). First line presents the original image. Columns present respectively an erosion, a dilation and a morphological gradient.

[7] J. Goutsias, H.J.A.M. Heijmans, and K. Sivakumar, "Morphological operators for image sequences," COMPUT VIS IMAGE UND, vol. 62 no. 3, pp. 326-346, 1995.

[8] J. Chanussot and P. Lambert, "Bit mixing paradigm for multivalued morphological filters," in International Conference on Image Processing and Its Applications, 1997, vol. 2, pp. $804-808$.

[9] O. Lezoray, C. Charrier, and A. Elmoataz, "Rank transformation and manifold learning for multivariate mathematical morphology," in EUSIPCO, 2009, pp. 35-39.

[10] E. Chevallier and J. Angulo, "The irregularity issue of total orders on metric spaces and its consequences for mathematical morphology," $J$ MATH IMAGING VIS, vol. 54, no. 3, pp. 344-357, 2016.

[11] E. Chevallier and J. Angulo, "Image adapted total ordering for mathematical morphology on multivariate images," in IEEE ICIP, 2014, pp. 2943-2947.

[12] M. A. Veganzones, M. Dalla Mura, G. Tochon, and J. Chanussot, "Binary partition trees-based spectral-spatial permutation ordering," in ISMM, 2015, pp. 434-445.

[13] O. Lezoray, C. Meurie, and A. Elmoataz, "Graph-based ordering scheme for color image filtering," IJIG, vol. 8, no. 3, pp. 473-493, July 2008.

[14] O. Lézoray, "Manifold-based mathematical morphology for graph signal editing of colored images and meshes," in IEEE SMC, 2016, pp. 109114.

[15] I. Ram, M. Elad, and I. Cohen, "Image processing using smooth ordering of its patches," IEEE T IMAGE PROCESS, vol. 22, no. 7, pp. 27642774, 2013.

[16] I. Ram, M. Elad, and I. Cohen, "Image denoising using nl-means via smooth patch ordering," in ICASSP, 2013, pp. 1350-1354.

[17] J. Ding, D. Han, J. Dezert, and Y. Yang, "A new hierarchical ranking aggregation method," in FUSION, 2016, pp. 1562-1569.

[18] C. Dwork, R. Kumar, M. Naor, and D. Sivakumar, "Rank aggregation methods for the web," in $W W W$. 2001, pp. 613-622, ACM.

[19] S. Velasco-Forero and J. Angulo, "On nonlocal mathematical morphology," in ISMM, 2013, pp. 219-230. 